Universidad de Ciencias Empresariales y Sociales, Buenos Aires, Argentina

Recibido: 30 de septiembre de 2013 Concepto de evaluación: 12 de enero de 2014 Aprobado: 17 de marzo de 2014

Artículo de reflexión DOI: http://dx.doi.org/10.14718/ revfinanzpolitecon.2014.6.1.5

(C) 2014 Universidad Católica de Colombia. Facultad de Ciencias Económicas y Administrativas. Todos los derechos reservados.

*Licenciada en Economía (UBA) con posgrado en Economías Latinoamericanas (CEPAL,

$U N)$, magíster (c) en Economía Aplicada (UCA). IECIF, Universidad de Ciencias Empresariales y Sociales, Buenos Aires, Argentina. Dirección de correspondencia: Arenales $2832-7^{\circ} \mathrm{F}$ (1425), Ciudad Autónoma de Buenos Aires Buenos Aires, Argentina. Correo electrónico: elianascialabba@gmail.com

**Licenciado en Economía (UBA), magíster en Finanzas Corporativas (UCEMA). IECIF, Universidad de Ciencias Empresariales y Sociales, Buenos Aires, Argentina. Dirección de correspondencia: Arenales $2832-7^{\circ} \mathrm{F}$ (1425), Ciudad Autónoma de Buenos Aires Buenos Aires, Argentina. Correo electrónico: lic.mrc@hotmail.com

\section{Recursos naturales y desarrollo local: el complejo oleaginoso argentino y la producción de biodiesel}

\section{RESUMEN}

Argentina cuenta con una gran cantidad de recursos naturales, específicamente los ligados al complejo oleaginoso. Los cambios en la demanda mundial y en los procesos de producción han dado lugar a la conformación de un complejo productivo en torno a ellos, el cual se encuentra en su etapa madura, y orienta su producción a la exportación, por lo que no solo se genera valor, sino también externalidades positivas, tanto en creación de empleo como en desarrollo sustentable. En este escenario, la producción de biodiesel en torno al complejo oleaginoso agrega valor a la producción, genera encadenamientos hacia atrás, adelante y hacia los lados, lo que da lugar a la creación de actividades complementarias.

Palabras clave: clústeres, desarrollo local, producción agrícola, agroindustria, biodiesel.

JEL: O13, O14, O44, Q13, Q16

\section{Natural Resources and Local Development: The Argentinian Oilseed Complex and Biofuel Production}

\section{ABSTRACT}

Argentina is very rich in natural resources, particularly those linked to the oilseed production. Global demand and improved means of production, have resulted in a mature and developed production infrastructure geared toward export. This generates not just revenue, but also has other positive effects, such as the creation of jobs and sustainable development. In this context, biofuel production adds multidimensional value to the vegetable oil industry and generations many spin-off industries.

Keyword: Clusters, local development, agricultural production, agroindustry, biofuel. 


\section{Recursos naturais e desenvolvimento local: o complexo oleaginoso argentino e a produção de biodiesel}

\section{RESUMO}

A Argentina conta com uma grande quantidade de recursos naturais, especificamente os ligados ao complexo oleaginoso. As mudanças na demanda mundial e nos processos de produção vêm dando lugar à conformação de um complexo produtivo em volta deles, o qual se encontra em sua etapa madura, e orienta sua produção à exportação, razão pela qual não somente se gera valor, mas também externalidades positivas, tanto em criação de emprego quanto em desenvolvimento sustentável. Nesse cenário, a produção de biodiesel em volta do complexo oleaginoso agrega valor à produção, gera encadeamentos para trás, adiante e para os lados, o que dá lugar à criação de atividades complementares.

Palavras-chave: clusters, desenvolvimento local, produção agrícola, agroindústria, biodiesel. 


\section{INTRODUCCIÓN}

El crecimiento y desarrollo económico ha sido una preocupación de los economistas de todos los tiempos. Ya los mercantilistas en su época consideraron que la prosperidad de una naciónEstado dependía del capital (para ellos, los metales preciosos) que pudieran acumular y los fisiócratas lo asociaban al producto neto, originado en la tierra, siendo los precursores de los estudios de la importancia de los recursos naturales en el proceso de expansión de una economía.

En los inicios de la economía como ciencia, Adam Smith, llamado el "padre de la economía" investigaba acerca de "la naturaleza y causas de la riqueza de las naciones" (1958 [1776]) y fue este autor el primero en plantear la existencia de rendimientos crecientes generados por la división del trabajo, idea que luego fue abandonada por su sucesor, David Ricardo (1973 [1817]), al presentar su teoría basada en los rendimientos decrecientes de la tierra, la cual fue extendida por los economistas neoclásicos a todos los factores productivos (Scialabba, 2012).

Más de un siglo después, Young (1928) retomó los postulados de Smith, planteando que el incremento de los retornos es una combinación de dos procesos diferentes: el primero se encuentra estrechamente ligado a la "función de progreso técnico", el segundo está relacionado con el crecimiento dado por la división del trabajo y los rendimientos crecientes. Sin embargo, no fue sino hasta a mediados del siglo XX, con el surgimiento de la teoría del desarrollo, que los rendimientos crecientes volvieron a estar en el centro de la teoría económica.

Myrdal (1956) planteaba que para iniciar el proceso de desarrollo era necesario que un factor especial pudiera generar una serie de movimientos económicos, los cuales generarían externalidades positivas, empujarían al mayor crecimiento y de esta forma se generaría una "causación circular y acumulativa". En tanto, en la visión de Hirschman (1961), opuesta a la del desarrollo balanceado de Lewis (1954), se planteaba que el mismo debía ser desbalanceado, a partir de la selección de sectores estratégicos por incentivar por parte del Estado, lo que daría comienzo al ciclo de desarrollo, el cual luego se "derramaría" al resto de los sectores económicos, y se generarían así eslabonamientos hacia adelante, atrás y hacia los lados.

Siguiendo estas recomendaciones, a partir de la década del 50, una gran parte de los países de América Latina se embarcaron en el proceso de sustitución de importaciones, y de esta manera daban protección a los sectores que deseaban incentivar. Es en este marco teórico donde la industria fue el sector estratégico seleccionado por los Estados como artífice del proceso de desarrollo. No obstante, este aumento de industrialización fue incompleto y no permitió que dichas economías se desarrollaran, debido a que la producción se canalizaba únicamente al mercado interno; por lo tanto, al no estar orientados a satisfacer a los mercados internacionales, no generaban la necesidad de ganar en competitividad. En palabras de O'Connor (2012b), "un país es competitivo en la medida en que produzca bienes y servicios que "superen" el examen de los mercados internacionales, al mismo tiempo que aumenta el ingreso de su población, es decir, satisface los requerimientos de equidad" (p. 64).

La congruencia de los malos resultados de la política proteccionista de industrialización sustitutiva de importaciones, junto a la década perdida de los años 80, dio lugar a la implementación del Consenso de Washington en los países de la región, el cual experimentó su peor fracaso en Argentina en 2002 con la caída del Plan de Convertibilidad, en el cual se habían seguido todas las recomendaciones del mismo. Sin embargo, a pesar del resultado final de la implementación de dicho consenso, la desregulación financiera y comercial, la liberalización de la cuenta capital y el plan de privatizaciones llevadas adelante en los años 90 dieron lugar a un fuerte proceso de modernización en lo referente a maquinaria y equipo del sector primario, especialmente del sector oleaginoso.

Este proceso se debió, en gran medida, al aumento de la superficie cultivable y de la 
productividad, generado por la utilización de nuevas tecnologías así como por la introducción de semillas transgénicas $R R$ (Round Up Ready de Monsanto), las cuales permitieron que el cultivo fuera explotado intensivamente y con mano de obra mínima, sumado a la incorporación de la siembra directa (técnica desarrollada por el Instituto Nacional de Tecnología Agropecuaria, INTA), como sistema de producción.

Así mismo, debe destacarse que el sector de oleaginosas representa el mayor generador singular de divisas y de ingresos para el sector agropecuario, en algunas zonas de la región pampeana se ha desplazado en forma relativa a otros cultivos de gran importancia, tales como el maíz y el girasol. Entre cuatro cultivos más importantes aportan el $90 \%$ de la producción nacional: trigo, maíz, soja y girasol. Debido a estos notables avances, en la actualidad el complejo oleaginoso argentino ha avanzado notablemente en el proceso de maduración, exhibiendo encadenamientos hacia atrás, hacia adelante y hacia los lados. En lo que a encadenamientos hacia adelante se refiere, hay una importante rama industrial que procesa el insumo agrícola (básicamente soja y girasol) y lo convierte en aceite y subproductos, duplicando así el valor por tonelada, en cuanto a la fase de comercialización que sigue, incluye servicios tan importantes como los de almacenamiento, transporte y embarque.

No obstante, según se observa, es necesario profundizar el proceso de industrialización vinculado al complejo productivo, con el fin de generar cadenas de alto valor agregado, incorporación de tecnología de punta y una mayor maduración del clúster. Es aquí donde la producción de biodiesel adquiere un rol preponderante: no solo es un producto con mucho valor agregado, con grandes potencialidades en cuanto a la conformación de encadenamientos productivos, sino que también, al tratarse de un biocombustible, es de vital importancia en vista de un desarrollo sustentable desde el punto de vista ambiental ${ }^{1}$, específicamente

1 Sin embargo, a pesar de la visión de desarrollo sustentable utilizada en el presente trabajo, es necesario mencionar la

Finanz. polit. econ., ISSN 2248-6046, Vol. 6, No. 1, enero - junio, 2014, pp. 95 - 114 Edición Especial - Economía Regional desde su "promoción como una alternativa para la mitigación de gases efecto invernadero (GEI) frente al uso de los combustibles fósiles, lo que permite a los países cumplir con los compromisos de reducción de GEl adquiridos bajo el Protocolo de Kyoto" (Dufey y Stange, 2011, pp. 13, 14).

En el presente documento se analizarán las características que deben verificarse en un territorio para la conformación de un clúster o complejo productivo y las experiencias exitosas a nivel mundial de clústeres en torno a recursos naturales, para luego presentar las etapas de maduración por las que transita el mismo y las actividades características que distinguen cada una de dichas fases.

A continuación se presentará el complejo oleaginoso argentino y se analizarán por separado los actores intervinientes en él y su interrelación: productores primarios, infraestructura y almacenaje, industria y canales de comercialización, y la fase de maduración en la que se encuentra el mismo. Se continuará con la descripción del procesos de producción de biodiesel, su legislación y marco regulatorio en Argentina, y datos de su evolución durante los últimos años. Por último, se presentarán las conclusiones a las que se arribarán al finalizar la investigación.

\section{CLÚSTERES EN TORNO A RECURSOS NATURALES: CARACTERÍSTICAS Y EXPERIENCIAS EXITOSAS}

Se entiende comúnmente por complejo productivo - o clúster- a una concentración sectorial y/o geográfica de empresas que se desempeñan en las mismas actividades o en actividades estrechamente relacionadas, tanto hacia atrás, hacia los proveedores de insumos y equipos, como hacia adelante y hacia los lados, hacia industrias procesadoras y usuarias, como a servicios y actividades estrechamente relacionadas con importantes y acumulativas economías externas, de aglomeración

existencia de teorías divergentes, como la que plantea que el monocultivo de la soja, principal insumo del biodiesel, produce un desgaste del suelo, y por lo tanto el desarrollo de las actividades generadas en torno a él no son sustentables. 
y especialización (por la presencia de productores, proveedores y mano de obra especializada y de servicios anexos específicos al sector) y con la posibilidad de llevar a cabo una acción conjunta en búsqueda de eficiencia colectiva.

Según Ferraro (2010), durante los últimos treinta años es posible observar un creciente interés en el estudio de este tipo de concentraciones y de los distintos elementos que intervienen en su desarrollo. Aunque los primeros aportes se remontan a Alfred Marshall y su "atmosfera industrial", a fines del siglo XIX, el tema comenzó a investigarse de manera sistemática a partir de la década de 1970. Los trabajos pioneros fueron realizados por autores que analizaron el éxito de varias localidades pertenecientes a la Tercera Italia. A partir de entonces se multiplicaron los estudios sobre diversas experiencias de desarrollo local, tratando de analizar los factores distintivos que contribuyeron a que tuvieran un mejor desempeño en términos relativos.

Para Marshall, los distritos son una concentración geográfica de empresas especializadas que, aprovechando la división del trabajo en fases productivas y la especialización sectorial, consiguen ventajas de escala frente a la gran firma integrada. Además, la cercanía física genera una dinámica innovadora: "la localización de la actividad productiva fomenta y educa la habilidad y la satisfacción, y difunde el conocimiento técnico. La presencia de agentes que se dedican a una misma actividad posibilita que éstos se eduquen mutuamente" (Marshall, 1890, p. 154).

En línea con la "atmosfera industrial" marshaIliana, Ferraro (2010) plantea que Becattini rescata estos conceptos, a fines de los años setenta, para estudiar el entonces reciente crecimiento económico en el centro y norte de Italia, basado en la dinámica de las pequeñas y medianas empresas. Sin embargo, supera la perspectiva exclusivamente económica al considerar al distrito como una entidad en la que se conjugan factores históricos, culturales y sociales. "La interpenetración y la sinergia entre la actividad productiva y la vida cotidiana es un rasgo dominante y característico del distrito en su funcionamiento" (Becattini, 1989, p. 6).
Es en este mismo contexto en el que Ramos (1998) plantea que la eficiencia del conjunto del complejo es mayor a la de cada empresa aisladamente, por las externalidades que genera cada empresa para las demás; es decir, la acción de cada empresa genera beneficios para sí misma como para las demás empresas del complejo. Para explicar la dinámica de dicha afirmación, el autor esgrime las siguientes cinco razones.

1. La concentración de empresas en una región atrae más clientes, y con esto el mercado se amplía para todas estas, más allá de lo que sería el caso si cada una estuviese operando aisladamente.

2. La fuerte competencia a la que da lugar esta concentración de empresas induce a una mayor especialización, división de trabajo $y$, por ende, mayor productividad.

3. La fuerte interacción entre productores, proveedores y usuarios facilita e induce un mayor aprendizaje productivo, tecnológico y de comercialización.

4. Las repetidas transacciones en proximidad con los mismos agentes económicos genera mayor confianza y reputación lo que redunda en menores costos de transacción.

5. La existencia del complejo, con conciencia de sí, facilita la acción colectiva del conjunto en pos de metas comunes (comercialización internacional, capacitación, centros de seguimiento y desarrollo tecnológico, campañas de normas de calidad, etc.).

Dadas estas características, el clúster más conocido en la actualidad es Silicon Valley en California. Además, existen numerosos ejemplos como los distritos industriales de Emilia Romagna (Italia) y Baden Wurttemberg (Alemania), la Ruta 128 (Estados Unidos), los complejos en torno a las industrias de computadores (Irlanda) y electrónica (Escocia) en los países desarrollados. 
En cuanto a los países en desarrollo, cabe mencionar la industria del calzado en Nuevo Hamburgo (Brasil), de electrónica y programas de computación en Bangalore (India), de instrumentos quirúrgicos simples en Sialkot (Pakistán) y de microelectrónica en Hsinchu Science Park (Provincia de Taiwán).

En línea con estas experiencias, es posible afirmar que dentro del complejo productivo las firmas son, por lo general, pequeñas y no elaboran productos finales completos, sino que cada una se especializa en una fase del proceso productivo, lo que implica una fuerte especialización productiva con una fuerte división social del trabajo. Por lo tanto, en este contexto es de vital importancia la coordinación entre dichas firmas.

Considerando como casos extremos el mercado y la integración, en los cuales un bajo costo de coordinación equivale a un alto costo de información y viceversa, se observa que los clústeres parecen tomar lo mejor de los dos mundos: bajos costos de coordinación y bajos costos de información, lo cual genera una muy significativa ventaja competitiva. Esta simultaneidad se obtiene de la estructura de transacciones de cuasi mercado, que caracteriza a los complejos productivos.

De esta forma, se generan relaciones de cuasi mercado que permiten explotar las ventajas de una alta división social del trabajo con bajos costos de coordinación y de información, están la confianza y la cooperación, incentivadas por la cercanía geográfica (Krugman, 1998).

Por otra parte, concentrando el estudio específicamente en los recursos naturales, a pesar de numerosas investigaciones que plantean la maldición de estos, también conocida como la "enfermedad holandesa" (Rodríguez y Sachs, 1999), existe evidencia de países con gran cantidad de los mismos que han articulado complejos productivos en torno a ellos, lo que ha creado clústeres de alto valor agregado (De la Torre, 2011; Cepal, 2005).

En palabras de Llach (1998), "los clústeres de este tipo se desarrollan normalmente con el paso del tiempo en regiones que tienen algún recurso natural en abundancia y comienzan normalmente con la explotación del recurso mismo, importando todos los insumos necesarios para tal fin y enviándolo en bruto a otra región (o país) para su procesamiento" (p. 8).

En el caso de los países con muchos recursos y escasa población, como Argentina, Australia o Canadá, en el comienzo de la explotación de los mismos no solo se importaban los insumos, sino que el mismo capital y como la mano de obra debían ser importados, además de la tecnología, las maquinarias y los demás insumos.

Caso emblemático de ello es el clúster en torno a la industria forestal en Finlandia, el cual es completo y profundo, ya que representa el $25 \%$ de las exportaciones del país. Según Ramos (1998), los múltiples e importantes encadenamientos con otras actividades que forman el clúster permiten agregar mucho valor a la fase extractiva y van elevando cada vez más la proporción de valor agregado gracias a actividades más complejas y que utilizan más conocimientos especializados.

También se observan los casos de clústeres maduros en Canadá y Australia, y más recientemente en Chile. Este último se concentra en tel complejo industrial que se da en torno a la minería, para lo cual el país tiene una fuerte ventaja comparativa; además, por la congráficación geográfica del país, donde la cordillera está cerca del mar, los costos de transporte a los mercados internacionales son relativamente más bajos que en otros países. Así mismo, se han conformado en el país trasandino clústeres para la industria vitivinícola, de frutas finas y del salmón.

\section{ETAPAS DE MADURACIÓN DE UN CLÚSTER}

En relación con los casos expuestos en la sección previa, Ramos (1998) plantea que un complejo basado en recursos naturales maduro pasa por cuatro etapas. En una primera fase, se extrae y exporta el recurso natural con el procesamiento local mínimo indispensable, dados los altos costos de transporte. Casi todo lo demás se importa: el grueso de los insumos, maquinaria e ingeniería. En una segunda fase, se ponen en marcha actividades 
de procesamiento y exportación y se comienzan a sustituir importaciones con producción local de algunos insumos y de equipos, y la provisión totalmente local de los servicios de ingeniería para la producción, y parcialmente local en lo que a diseño se refiere. En la tercera fase se comienzan a exportar algunos de los bienes y servicios que primeramente se sustituyeron -insumos y maquinarias básicas- a mercados poco exigentes; la ingeniería es casi totalmente nacional, y se profundiza la exportación de productos procesados cada vez más sofisticados. Finalmente, en la última fase se exporta de todo: productos procesados de gran variedad y complejidad, insumos y maquinaria a mercados exigentes, servicios de ingeniería de diseño y consultarías especializadas. Así mismo, las empresas del país comienzan a invertir en el exterior en ese mismo rubro.

No obstante, Pietrobelli y Rabelloti (2005) plantean que los orígenes de las concentraciones empresariales de este tipo pueden tener distintos puntos de partida y diferentes dinámicas de evolución. En el caso de la industria salmonera de Chile, la misma comenzó con una cooperación públicoprivada y con un proceso de aprendizaje colectivo cuyo catalizador fue el aumento de la demanda externa y una política activa destinada a apoyar las exportaciones, mientras que la transformación de la economía local de Petrolina-Juazeiro (Brasil), en un dinámico complejo productor de mango y uva, fue el resultado de una planificación gubernamental centralizada, y la creación de la aglomeración productiva de manzanas en Santa Catarina (Brasil) fue producto de la iniciativa de empresarios pioneros sumada al apoyo y las políticas de extensión del sector público.

Estos tres casos presentados son solo algunos de los exitosos de aglomeraciones en torno a los recursos naturales; sin embargo, dan muestra de que si bien una vez comenzado el proceso de producción este tiende a presentar distintas fases de desarrollo, estas de ninguna manera son lineales, y dependen claramente del punto de partida.

A todo esto, es necesario añadir que la organización interna de la articulación productiva debe poder compatibilizar los procesos de cooperación, colaboración y competencia entre agentes independientes. Ya que los resultados obtenidos refuerzan las ventajas de las firmas, deberían incrementar las posibilidades de penetración en mercados, la capacidad competitiva, la productividad y las tasas de beneficios. Las cualidades de las formas de interacción de los agentes permiten generar procesos simultáneos de innovación, aprendizaje y desarrollo de ventajas competitivas, algunos en el ámbito microeconómico y otros a nivel colectivo (Ferraro, 2010).

En efecto, sin la acumulación de progreso tecnológico la evolución del complejo se frenará, lo cual se limitaría a la "renta pura" de la fase extractiva. Es aquí donde la innovación juega un rol preponderante, y generalmente en los países que conforman complejos productivos en torno a recursos naturales se comienzan dichos procesos a través de desarrollos de "imitación" (Fagerberg, 2003; Fagerberg y Verspagen, 2001), en los cuales el progreso técnico genera mayores economías externas y potencia las ya existentes (Kaldor, 1981). En este contexto, las innovaciones en este tipo de firmas es incremental, y están basadas en un learning by doing and by using y tienen un alto contenido adaptativo. Además, el grado de formalización e institucionalización de la investigación tecnológica, en comparación con grandes empresas, es bajo. Así pues, esta caracterización de la conducta tecnológica de los complejos productivos es relevante, ya que aportes posteriores en la literatura plantean que la supervivencia de los mismos en el marco de la globalización requiere cambios en esta modalidad de comportamiento.

\section{COMPLEJO OLEAGINOSO EN ARGENTINA}

El complejo oleaginoso, y la soja en particular, se ha convertido en uno de los protagonistas del devenir económico de Argentina en los últimos años. En una economía de gran inestabilidad de sus mayores variables macroeconómicas (inversión, sector fiscal y externo) y estancamiento relativo del producto 
en el largo plazo, el complejo oleaginoso es uno de los pocos que recorre una senda de expansión continua desde hace ya más de tres décadas.

Comenzando el análisis del clúster oleaginoso, se observa que las industrias proveedoras de insumos están controladas por grandes empresas transnacionales, con fuertes reestructuraciones intrasectoriales y un rápido proceso de innovación ${ }^{2}$. La etapa siguiente, la primaria, está caracterizada por las fuertes tendencias al incremento del tamaño de las explotaciones, lo que genera economías de escala con mejor aprovechamiento de la superficie cultivada, así como la difusión de las modificaciones genéticas realizadas en las semillas de la soja, lo que da lugar a una "doble" expansión de la producción en esta etapa.

La evolución de la producción de soja, luego de la incorporación de las modificaciones genéticas de las semillas, se ha incrementado desde 1993. Solo exhibe fuertes desaceleraciones en las campañas 1997-1998 y 2008-2009. Así mismo, en la última campaña con información disponible (2011) se verificó una contracción del 7\%, explicado principalmente por cuestiones climáticas, aunque se observa que la producción mantiene su tendencia ascendente. Por otra parte, la fuerte suba de los precios de los productos oleaginosos, especialmente de la soja, hizo que la participación de este último cultivo en la producción total del complejo se incrementara año a año.

En la campaña 1993-1994, la relación soja/ resto de los cultivos era del $77 \%-23 \%$. La misma disminuyó hasta la campaña 1997-1998, en la cual la relación era de $62 \%-38 \%$. A partir de ese año, la participación de la producción de soja en el total del complejo oleaginoso comenzó a incrementarse de la mano de la suba de los precios internacionales. El máximo se presentó en la campaña 2004-2005,

2 Estos procesos también afectan a las industrias proveedoras de maquinarias y equipo para el agro y se enmarcan en la teoría evolucionista, más conocida como neoschumpeteriana, donde se tratan tres componentes que determinan el proceso de innovación, y actúa de forma similar al proceso evolutivo en las ciencias naturales: heterogeneidad de los agentes, mutación para adaptarse a los mercados globalizados y selección de las que mejor se inserten en dichos mercados. cuando la producción de soja representó el $98 \%$ de la producción oleaginosa. En la última campaña con datos disponibles, la soja tuvo una participación del $92 \%$, y se espera que la tendencia siga por encima del $90 \%$.

En este escenario, es importante destacar la atomización que se verifica en la producción primaria conformada por un gran número de productores, mientras que se va concentrando la actividad a medida que se avanza en los procesos de industrialización y en la etapa de exportación. En la etapa de acopio, las funciones están dadas por la compra, almacenaje y distribución de la producción, en la que intervienen una gran cantidad de industrias e intermediarios, y no solo se limitan a sus funciones tradicionales, sino que también se encargan de extender el financiamiento para que se introduzcan mejoras tecnológicas.

A continuación, se presenta la etapa correspondiente al almacenaje, con importantes innovaciones gracias a la difusión de silo-bolsa, en la que los grandes exportadores pueden mantener su producción almacenada con gran flexibilidad. Debe destacarse que antes de la implementación de estos cambios, dicha actividad se encontraba a cargo del sector público, y a partir de la década de los 90 con la etapa de las privatizaciones pasó a cargo entidades privadas.

Luego, aparece el proceso industrial en el complejo productivo. En la primera industrialización, la producción de aceite crudo de soja está asociada tecnológicamente a la de girasol, por lo que pueden producirse en la misma planta. Los márgenes que se obtienen en la comercialización internacional impulsan de forma sostenida aumentos en las escalas de las plantas procesadoras, y esfuerzos tendientes al logro de mayores eficiencias. Esta tendencia se impone en los principales países productores, lo cual convierte la escala en una importante barrera a la entrada de nuevas firmas.

El sector presenta desde hace varios años un gran dinamismo, con importantes inversiones tendientes a ampliar su capacidad de molturación y almacenamiento y a mejorar las instalaciones portuarias. La industria aceitera nacional, dotada de 
alta tecnología, registra el mayor crecimiento entre los principales productores del mundo. Por otra parte, las actividades logísticas y de infraestructura de transporte y almacenaje se han ido integrando a los grandes traders y empresas de la molienda, a partir de las privatizaciones y la desregulación de los mercados en la década de los 90 en el país.

Por último, la comercialización externa está controlada a escala mundial por grandes traders del comercio de granos y oleaginosas y por grandes empresas multinacionales de la trituración, cuyas filiales dominan esta etapa en el mercado local. En los mercados internos, los canales de comercialización mayorista y minoristas están concentrándose crecientemente en manos de las grandes cadenas minoristas de la alimentación, o gran distribución minorista.

En relación con el perfil exportador, las colocaciones externas de aceites tienen un peso decisivo. A partir del desarrollo conjunto de un entramado empresarial e institucional público y privado centrado en la difusión horizontal de innovaciones organizativas, de información sobre mercados humanos y significativas inversiones privadas y públicas, se desenvuelve un importante número de pymes dinámicas orientadas al mercado interno y externo.

Todos estos factores permitieron a la industria incrementar los volúmenes procesados y mantener el liderazgo mundial en las exportaciones.

\section{Principales actores de la producción}

La interdependencia de todos los actores de la cadena y las industrias y pymes relacionadas definen al poderoso "clúster oleaginoso" en toda su magnitud. Como surge de numerosos estudios, es posible afirmar que el complejo productivo oleaginoso es uno de los subsistemas agroalimentarios más importantes de Argentina, particularmente de la provincia de Santa Fe. Su fuerte dinámica exportadora y la expansión registrada en la última década, motorizadas por demandas en aumento, importantes cambios tecnológicos y transformaciones en los marcos regulatorios internos e internacionales, ubican a las exportaciones de este complejo a la cabeza de las exportaciones del país.

La identificación de quiénes y cómo lideran estos procesos dentro de cada una de las tramas productivas más dinámicas es esencial para analizar el aporte de estas formas organizativas al proceso local de acumulación.

En el análisis de la particular dinámica presentada por el clúster, se estudiarán exhaustivamente los actores de la producción primaria, la infraestructura y almacenaje, la transformación industrial y los canales de comercialización, y la interdependencia existente entre estos principales actores del proceso de producción del complejo.

\section{La producción primaria}

En la actividad primaria se destaca la incorporación de la siembra directa como sistema de producción y toda la incorporación tecnológica que la acompaña (maquinaria, agroquímicos, semilla). La nueva técnica consiste en sembrar sin necesidad de roturar la tierra ni de llevar a cabo los procesos asociados, como el emparejado y las labores posteriores de cobertura. En lugar de ello, se recurre a un procedimiento único: se abre un surco donde se depositan la semilla y eventualmente los fertilizantes, y luego se lo aplasta (Bisang, 2003).

En el caso de la soja, la transición de la soja convencional a la genéticamente modificada supuso una profunda transformación de la organización productiva y de las instituciones del sector agrícola argentino. En la actualidad predominan la "siembra directa" (cero labranza) y la "agricultura de contratos", y el agricultor tradicional cumple un papel mucho menor como agente responsable de organizar la producción. Dicho papel ahora está a cargo de grandes empresas y de subcontratistas de ingeniería agrícola, de carácter independiente, que atienden sucesivamente distintos predios y organizan la producción. Uno de los cambios más significativos fue el aumento de la participación del gasto en agroquímicos a partir de la segunda mitad de la década de los 80 . El mayor consumo de agroquímicos se produjo por la disminución de 
los precios de estos como consecuencia de la apertura económica. Esta incorporación tecnológica motivó un crecimiento en la demanda de servicios vinculados con la contratación de máquinas, fletes, consultorías agrotécnicas, etc.

Actualmente se firman contratos de riesgo para el financiamiento y la organización productiva con intermediarios financieros y bancos especializados en el financiamiento de la producción de soja. El paquete tecnológico — semillas, abonos, herbicidas - utilizado por los subcontratistas es propiedad privada de grandes empresas transnacionales, como Monsanto, Cargill y otras, lo que establece una marcada diferencia con el patrón predominante en la revolución verde de la década de 1960, cuando la tecnología agrícola era fundamentalmente un bien público distribuido por organismos agrícolas del Estado.

El tema relevante en materia de difusión del cultivo es básicamente cuánto más puede seguir expandiéndose esta oleaginosa en Argentina y en detrimento de qué productos, si no es como una expansión genuina del horizonte agrícola, y al mismo tiempo si es sustentable esta fuerte tendencia al monocultivo que se presenta desde la faz de los recursos naturales y del contexto económico.

\section{Infraestructura y almacenaje}

En términos de infraestructura, se reconoce la importancia de la logística, por la Hidrovía y el corredor bioceánico, que dan otro dinamismo a la principal ciudad de la provincia de Santa Fe, pero se genera un cuello de botella en los puertos y la Hidrovía, pues se concentra más del $80 \%$ de lo que se exporta en la Argentina.

Por otra parte, se pondera la estructura fuertemente amparada en pequeñas y medianas empresas. También debe mencionarse la intervención estatal para crear condiciones favorables para el desarrollo de las industrias. La terminal portuaria de Dreyfus sobre el Río Paraná tiene un calado adecuado para la operación de grandes buques de ultramar y su capacidad de embarque es de 2800 toneladas por hora base de cereales y de 1000 toneladas por hora de aceites, pudiendo recibir hasta 35.000 toneladas de granos por día en camiones, barcazas y ferrocarril.

El área portuaria consta de un muelle cerealero, un muelle de barcazas que se encuentra en operación desde el 29 de octubre de 1998 y el muelle aceitero. El muelle para embarque de aceite, que está ubicado en el km 396 del Río Paraná, consiste en un segundo sitio de atraque para embarque de aceites vegetales. Las instalaciones se han calculado con base en un tipo de buque aceitero, con un porte bruto de 50.000 toneladas y un desplazamiento de 60.000 toneladas, eslora de 220 metros y manga de 32 metros, y un calado máximo de 11,4 metros. Este muelle de embarque surge como una necesidad de aumentar las funciones del puerto, en virtud del incremento comercial de las exportaciones y con el fin de mejorar la logística operativa.

En Argentina más del $80 \%$ del transporte de granos se realiza en camión; solamente un pequeño porcentaje se transporta por ferrocarril ${ }^{3}$. En la década del 40, este era el principal medio en la comercialización de granos, pero su participación fue disminuyendo continuamente hasta principios de la década del 90. Desde la concesión a operadores privados realizada en 1991-1992 los volúmenes transportados se incrementaron moderadamente, y se estabilizaron hacia fines de la década.

Un aumento en la participación del ferrocarril producto de las inversiones en el sector llevaría a una reducción en el costo de transporte ${ }^{4}$, principalmente de las regiones más alejadas de las industrias. Es por esto que la etapa de almacenaje y la de transporte son hoy -luego de las inversiones en nuevas plantas aceiteras y puertos- los dos eslabones más débiles de la cadena.

3 Algunos de los grupos concesionarios de vías férreas están constituidos por empresas del sector aceitero, de modo que estas transportan la materia prima hasta sus plantas y la producción obtenida a los puertos, con lo cual se logra una mayor integración en la cadena.

$4 \quad$ Las plantas procesadoras se aprovisionan de soja en un radio de menos de $300 \mathrm{~km}$, lo que significa un reducido costo de flete. 


\section{Transformación industrial}

El estudio de la conducta innovativa de las empresas agroindustriales presupone introducir un marco teórico relacionado con el estudio de la innovación. El obstáculo más importante que encuentra el capital es el de la renta de la tierra. Toda vez que se han ocupado las tierras más fértiles, el capital puede avanzar hacia nuevas tierras marginales o profundizar su inversión en las tierras más fértiles ya en producción, o ambos caminos, aunque en general no a través de los mismos actores ni de las mismas condiciones.

El progreso técnico es la forma como el capital profundiza su inserción en las tierras más fértiles y esto se traduce en una elevación de la composición del capital expresado en medios de producción como las maquinarias, los agroquímicos, los fertilizantes. La intensificación de la producción lleva a que la tierra deje de ser el medio de producción fundamental y el capital expresado en maquinarias y agroquímicos pase a ocupar un papel primordial y dinámico.

Una de las principales industrias que surgen del complejo oleaginoso es la aceitera. Las características que presenta esta industria son los altos niveles de capacidad de las fábricas, una alta concentración del mercado en pocas empresas, la regionalización en la ubicación de las empresas y una gran participación de firmas extranjeras en el mercado argentino. El mismo cuadro de grandes cambios económicos e institucionales se encuentra al examinar la producción de aceite vegetal a partir de la soja genéticamente modificada. En la década del 90 se instalaron nuevas plantas de última generación, instalaciones de producción catalíticas altamente automatizadas, en las cuales la productividad laboral es diez veces mayor que la que se lograba con la tecnología manufacturera del decenio de 1970, que predominaba en la Argentina hasta hace relativamente poco tiempo.

En el contexto analizado, puede concluirse que la producción de soja genéticamente modificada y aceite vegetal en Argentina trajo aparejados grandes cambios en las instituciones, la tecnología de producción y la organización industrial. Han surgido en la economía mayores rendimientos a escala, mayores externalidades y muchas capacidades tecnológicas nuevas.

\section{Canales de comercialización}

Las empresas que procesan y exportan este tipo de productos suelen estar integradas también con la exportación de materia prima y en una gran medida disponen de la logística de embarques (almacenamiento, acondicionamiento, puertos, etc.) -incluso en algunos casos el propio "charteo" de los buques-, la cual le permite integrar el negocio y amortiguar en muchas oportunidades ciertas pérdidas específicas de la operación de molienda.

Los productores primarios son, en su gran mayoría, no integrados y venden la totalidad de su cosecha a los exportadores o a los industriales. Hay muy pocos productores integrados que realizan el proceso completo, desde la producción primaria hasta la comercialización de su propia producción. Los industriales tienen políticas de asistencia al productor, que consisten en proporcionar los insumos respectivos y asesoramiento sobre el cultivo, recibiendo en concepto de pago determinados volúmenes del producto cosechado. Esta modalidad básicamente ocurre en zonas cercanas a las plantas industrializadoras

Los compromisos contraídos con el exterior muchas veces, y a pesar de la baja o nula rentabilidad del negocio, obligan a muchos operadores a continuar trabajando para satisfacer dichas necesidades.

\section{Producción e inversiones}

La expansión productiva registrada durante la década del 90 estuvo sustentada en la difusión y adaptación de innovaciones tecnológicas, el surgimiento o consolidación de grupos acotados de grandes empresas en las etapas principales de las tramas productivas que articulan esas producciones y una clara inserción en los mercados externos. Estos factores imprimieron un marcado dinamismo 
a las tramas y contribuyeron a modelar sus principales rasgos técnicos y productivos. Así, las tramas productivas aparecen como formas de organización impulsoras del crecimiento económico logrado en estas décadas, por su presunta capacidad de generar competitividad genuina.

Se produce un cambio hacia la agricultura continua, cuyas principales características han sido las siguientes:

1. Una mayor extensión de la etapa agrícola de la rotación.

2. Roturación de pastizales para pasarlos a agricultura continua.

3. Mayor intensificación en el uso de insumos, especialmente herbicidas e insecticidas.

4. Aumento de la capacidad de uso de la maquinaria agrícola, especialmente tractores y sembradoras.

5. Incremento sustancial del ciclo agrícola y extracción de cosechas (tres cosechas/ dos años).

6. Aumento de la escala de producción.

7. Incremento de la frontera agropecuaria, directamente con agricultura.

Por otra parte, también ha habido en los últimos años cambios de significación en el perfil de la oferta, en medio de un rápido proceso de actualización innovadora. Las mejoras en las semillas (introducción de transgénicas y otras técnicas) van perfilando un nuevo paradigma tecnológico, que diversos autores llaman la "agricultura dominada por el conocimiento".

Esa adopción permanente de tecnología por parte de los productores es complementada desde mediados de la década de los 90 , con el uso de variedades modificadas genéticamente resistentes a herbicidas como el glifosato, que permitieron en forma conjunta con el nuevo esquema de labores disminuir fuertemente los costos de implantación de este cultivo y, según sus seguidores, dar un marco de sustentabilidad al sistema productivo, para evitar así los nocivos efectos de la erosión de los suelos.
El desarrollo de una agricultura basada en la centralidad del cultivo de soja, en la disponibilidad de tecnología de insumos y en la inversión en maquinarias especializadas implicó una incorporación constante de capital que permitió el incremento de la superficie trabajada. A la hora de invertir en maquinarias, independientemente del estrato y el tipo de tenencia, la elección más señalada fue la sembradora de siembra directa. Así como esta sembradora es una inversión considerada necesaria en todos los estratos y tipo de tenencia, la inversión en pulverizadora y cosechadora ${ }^{5}$ responde más a la necesidad de ser competitivos para la toma de tierra, y por lo tanto se halla más presente entre los propietarios arrendatarios.

La decisión de incorporar una determinada maquinaria no se vincula directamente al aumento de productividad. La inversión, orientada al aumento del ingreso, puede relacionarse con la ocupación de mano de obra familiar (permitiendo que algún miembro trabaje como contratista de servicios) y/o a tener mayores posibilidades de competir en el mercado de alquiler de tierras. La inversión en maquinaria no garantiza en sí misma un aumento de productividad pero sí de producción, aspecto que posibilita a menor costo y tiempo ampliar la superficie trabajada.

Entonces, en el área de las innovaciones tecnológicas incorporadas a la maquinaria agrícola, herramientas, equipos y aparatos empleados en las diversas subactividades del sector agropecuario, se ha podido apreciar una verdadera revolución tecnológica durante los años de la posconvertibilidad. Las causas principales de este fenómeno fueron las siguientes:

1. La gran oferta generalizada de maquinarias e implementos que los fabricantes pusieron en el mercado como respuesta a las pautas de competitividad y a la apertura

$5 \quad$ Las cosechadoras constituyen una elevada inversión económica y normalmente se las utiliza no sólo en la superficie operada sino también en campo de otros productores en los que se presta el servicio de cosecha. Los limitantes en la posibilidad de capitalización en los ' 90 explicarían el estancamiento en la compra de cosechadoras. 
de la economía. El progreso tecnológico de los equipos de origen nacional.

2. La proliferación de marcas, niveles de calidad y variedades de equipos - con especificaciones técnicas acordes a los distintos usos - que fueron capaces de ir satisfaciendo una demanda cada vez más exigente.

3. El descubrimiento de nuevos equipos para responder a las necesidades de los productores.

4. La introducción de la computación en la maquinaria y de sensores para realizar monitoreos durante el desarrollo de las labores. Este fenómeno se ha llamado la "agricultura de precisión".

5. Las facilidades crediticias debido a la estabilidad que alentaron la demanda y también las inversiones de las empresas en nuevas tecnologías; pero principalmente esto fue la consecuencia de la apertura de nuestra economía a la competencia externa proveniente de la industria brasilera y del resto de los países industrializados.

\section{PRODUCCIÓN DE BIODIESEL}

El biodiesel es un biocombustible líquido que se obtiene a partir de lípidos naturales como aceites vegetales o grasas animales, con o sin uso previo, mediante procesos industriales de esterificación y transesterificación, y que se aplica en la preparación de sustitutos totales o parciales de naftas y gasolinas, obtenidas del petróleo.

Es la sustitución del petróleo lo que hace tan deseable la producción del biodiesel, ya que su uso genera menor contaminación y otorga, al menos parcialmente, mayor seguridad energética. Sin embargo, debe considerarse que su producción y comercialización masiva también tienen fuertes impactos ambientales y consecuencias sociales, pues generan un aumento de la deforestación de bosques nativos, la expansión indiscriminada de la frontera agrícola, el desplazamiento de cultivos alimentarios y para la ganadería, la destrucción del ecosistema y la biodiversidad, y el desplazamiento de los trabajadores rurales. Por esta razón, es necesaria una fuerte intervención estatal con el fin de regular $y$, en caso de ser necesario, mitigar, dichos impactos. No obstante, es menester destacar que debido a la naturaleza estratégica del biodiesel, la viabilidad de la industria depende de los costos de producción, debido a que se trata de un mercado creado por políticas gubernamentales, plasmadas en ambiciosas metas de penetración y generosos incentivos fiscales y no por las fuerzas propias del mercado.

Según un estudio de Cepal realizado por Dufey y Stange (2011), para los biocombustibles de primera generación el cultivo energético es el ítem de costos más significativo. En el caso del biodiesel con base en cultivos templados, como las oleaginosas, el costo del feedstock representa el $90 \%$ de los costos totales. Esto significa que los cambios en los costos de los cultivos energéticos tienen una fuerte incidencia en la rentabilidad de la industria.

En Argentina, a diferencia del resto de los países productores de biocombustibles que lo producen por temas estratégicos, el principal desarrollo del mismo está ligado a la existencia de ventajas comparativas en la producción de aceite de soja y no al establecimiento de un régimen de protección al sector. Es por eso que la producción de biodiesel en torno al complejo productivo oleaginoso argentino agrega valor a la producción del clúster, lo cual genera encadenamientos hacia atrás y adelante, como hacia los lados, y esto origina nuevas oportunidades para actividades complementarias.

De esta forma, el clúster estudiado en el presente trabajo, el cual en su etapa madura debe orientar su producción a la exportación, no solo genera valor, sino que provoca externalidades positivas, tanto en la creación de empleo como en desarrollo rural y local.

En torno a dicha temática, según Comisión de Energía de Naciones Unidas (UN-Energy), una de las principales motivaciones del desarrollo de los biocombustibles son los impactos positivos que 
estos puedan tener sobre el desarrollo rural y local. El nivel de mano de obra utilizada en la producción de dichos combustibles es más intensivo en comparación con aquel de otras fuentes tradicionales de energía. De esta forma, es de esperarse que esta industria genere más empleos por unidad de energía que aquella de los combustibles petroquímicos e hidro-energéticos (UN-Energy, 2007). Así mismo, el empleo asociado a dicha producción se vincula con trabajos más estables y mejores beneficios en relación con otras actividades agrícolas. No obstante, es necesario considerar que existe la preocupación que la expansión de la producción de biocombustibles puede generar, o exacerbar, malas prácticas laborales, en las producciones a pequeña y gran escala.

Reforzando el estudio acerca del impacto sobre el desarrollo local, la producción de biocombustibles genera fuertes impulsos sobre la economía rural y local cuando se produce para el consumo interno y se incluye a pequeños agricultores. Sin embargo, se espera que los efectos dinamizadores de los biocombustibles sean mayores cuando los productores, empresarios y empleados de la industria inviertan y/o gasten localmente sus ganancias, incluso a través de impuestos locales (UN-Energy, 2007).

Por otra parte, los subproductos y coproductos del procesamiento de los biocombustibles como la glicerina, alimentos de alto valor proteico para el ganado y fertilizantes, también podrían aumentar los ingresos locales (Worldwatch Institute, 2006). En tanto, otra forma de aumentar la economía local es incluir los biocombustibles como insumo de las actividades agrícolas, tanto en maquinarias, como en tractores o aviones para tareas de fumigación, etc.

Desde el punto de vista social, una gran parte de las comunidades pobres, especialmente aquellas en zonas rurales y aisladas, no tienen acceso a servicios eléctricos o energéticos básicos, lo cual disminuye sus oportunidades de desarrollarse económicamente y mejorar sus estándares de vida. Así, el uso de biocombustibles aumenta la accesibilidad de estas comunidades a la energía.
En el caso de localidades remotas, los programas descentralizados a pequeña escala en localidades rurales son una buena alternativa frente al uso de combustibles fósiles - de alto precio- para abastecer las redes eléctricas que proveen a hogares y pequeñas empresas (Dufey y Stange, 2011).

\section{Antecedentes y marco regulatorio en Argentina}

Desde 2007, Argentina comenzó a producir y a exportar biodiesel a partir de productos agrícolas. El feedstock de preferencia en este tipo de biocombustibles es el cultivo de soja, y en el caso argentino el $100 \%$ de la producción proviene de este. Si bien el proceso de investigación y desarrollo en el mundo comenzó mucho tiempo antes como consecuencia del aumento del precio del petróleo en la década del 70, en Argentina este recién comenzó a desarrollarse a partir de 2000.

A pesar de que el desarrollo comenzó a llevarse a cabo a principios de la década, la producción de biodiesel empezó a regularse a partir de 2009 cuando se reglamentó la Ley 2633 de los Bosques Nativos, la cual fue aprobada en 2007 y que en consecuencia delimitó las áreas de siembra y de bosques nativos. En este marco regulatorio, la producción de biodiesel en Argentina creció significativamente e incluso las exportaciones se incrementaron de manera sostenida desde 2007.

Además de la anteriormente citada, se aprobó la Ley 26093, reglamentada por el Decreto 109 de 2007, el 13 de noviembre de 2008, la cual creó un régimen de promoción para los biocombustibles y fue modificada por la Ley 26334. La medida más importante que establece esta ley es el uso de combustibles fósiles con una proporción de mínimo un $5 \%$ de biocombustibles (B5) - medido sobre la cantidad total del producto final- a partir de 2010, y dejó abierta la posibilidad de que el corte vaya creciendo a una mayor proporción. Esta ley también establece beneficios impositivos importantes con estabilidad fiscal, y facilita así la canalización de inversiones. Este régimen de inversiones opera a través del otorgamiento de cupos fiscales, 
que los fabricantes de biocombustibles utilizarán como licencia de operación. El cupo fiscal genera el derecho a quien lo obtenga de colocar toda su producción en las compañías petroleras obligadas a la incorporación de biocombustibles en la nafta y el gasoil que expendan.

En agosto de 2007, se verificó un cambio en la legislación existente, pasando de ser el mínimo un $5 \%$ a un $7 \%$ (B7), y en julio de 2012 hubo una modificación en la legislación que establecía el uso de combustibles fósiles con una proporción de al menos un $7 \%$ de biocombustibles (B7), y se la reemplazó por otra que elevó a un 10 \% la proporción de biodiesel en los combustibles (B10). El Gobierno sigue estudiando el hecho de incrementar el corte hasta un $20 \%$ (B20), máximo tolerado por la tecnología actual (motores diesel).

\section{Producción y exportación de biodiesel}

Como se mencionó previamente, la producción de biodiesel en Argentina es apuntalada por la legislación vigente, la cual con cada modificación incrementa la cantidad de biocombustible mínimo que deben contener los combustibles fósiles. El aumento sostenido de la producción de este bien se encuentra cimentado entonces en dos pilares: la exigencia gubernamental, es decir, una política pública activa, sobre el biodiesel, junto con la creciente demanda internacional, dado que el principal destino de este producto es el mercado externo.

Por otro lado, el dilema que se les presenta a los productores es comercializar la soja sin que esta pase por un proceso industrial, debido a los altos valores de esta en el mercado mundial, o transformarla en biodiesel y agregar valor a la producción, aun a costa de obtener una menor rentabilidad. En el caso argentino este dilema es crucial, debido a que el costo del aceite representa aproximadamente el $88 \%$ del costo total, en línea con las cifras presentadas por Dufey y Stange (2011), por lo que tener acceso a volúmenes grandes con una calidad adecuada y con un precio competitivo resulta fundamental para asegurar el resultado económico en esta agroindustria.
El biodiesel argentino está elaborado sobre la base de aceite de soja, y la industria que lo elabora está instalada junto al complejo aceitero de la provincia de Santa Fe, que representa el $75 \%$ de la capacidad instalada. La siguen Buenos Aires $(10,3 \%)$, Santiago del Estero (6,9\%), San Luis (3,9\%), Neuquén $(2,8 \%)$ y Entre Ríos $(0,4 \%)$.

Si bien el perfil de la industria es mayoritariamente exportador, hay varias empresas interesadas en sumarse a la comercialización en el mercado interno. Analizando con profundidad el mercado de biodiesel, se observa que el mismo presenta un crecimiento sostenido como consecuencia del aumento de la demanda de combustibles, tanto a nivel interno, como externo.

Por otra parte, aprobado el Régimen de Promoción de la Producción de Biocombustibles se verifica que la producción de biodiesel creció de 113.182 toneladas producidas en diciembre de 2009 a 117.314 producidas en marzo de 2013, con un pico máximo en la producción de 257.933 toneladas alcanzadas en enero de 2012. En tanto, las exportaciones presentaron un comportamiento similar. En diciembre de 2009 alcanzaron las 121.891 toneladas, y en marzo de 2013 se ubicaron por debajo del valor inicial de la serie, sumando 89.700 toneladas. En octubre de 2011, las exportaciones llegaron a su máximo, sumando 196.262 toneladas.

De esta forma, Argentina es el segundo exportador mundial de biodiesel, con una participación del 13,1\% del mercado, luego de Estados Unidos que exporta el 14,3\% de la producción, aunque es el primer exportador neto, alcanzando los 1300 millones de litros en 2010, seguido por Estados Unidos que enviaron (neto) al mercado externo 600 millones de litros.

El incremento en los volúmenes de producción que mostró el complejo agroindustrial del biodiesel tras la aplicación de la legislación analizada en la sección previa se explica por dos razones básicas:

1. La alta eficiencia que presenta la cadena agroindustrial de soja, que incluye 
aspectos relacionados con su tecnología agrícola de producción, las distancias relativamente cortas entre la principal zona de producción y el complejo agroindustrial aceitero, la escala grande y tecnología moderna que utilizan las plantas aceiteras y la facilidad de exportar esa producción mediante transporte marítimo.

2. Un incentivo importante desde el punto de vista impositivo, ya que las exportaciones de aceite de soja deben tributar en concepto de derechos de exportación (retenciones) un $32 \%$ de su valor, mientras que el biodiesel en un principio tributaba $5 \%$ (Decreto 509 de 2007) y luego se incrementó a $20 \%$ (Resolución 126 de 2008). Esta señal resultó determinante para que la industria aceitera se involucrara activamente en la elaboración y exportación de biodiesel.

En cuanto a las exportaciones de dicho combustible es necesario mencionar que actualmente el $75 \%$ de las plantas se ubican en la provincia de Santa $\mathrm{Fe}$, especialmente en las localidades de Puerto General San Martín, San Lorenzo y Rosario. Estas localidades forman el principal polo aceitero del país y cuentan con una infraestructura diseñada específicamente para exportar a través de la Hidrovía Paraná-Uruguay, lo que implica la existencia del clúster más grande a nivel mundial en el sector de las oleaginosas. Esta concentración hace que las distancias desde la zona de producción hasta los puertos de embarque sean, en promedio, de unos 300 kilómetros y de ahí una vez producidas, la distancia al puerto es insignificante, lo que genera menores costos de comercialización.

Sin embargo, medidas aplicadas por la Unión Europea, principal importador neto de biodiesel procedente de Argentina, han generado una contracción en los envíos al exterior en los últimos meses. Como muestra de esto se observa la caída en la producción, orientada principalmente al mercado externo, a partir de finales de 2012.
Estas restricciones al biodiesel proveniente de Argentina tienen su origen en la expropiación de Repsol YPF a España realizada por el Gobierno nacional el 3 de mayo del pasado año. Así mismo, en solidaridad a la situación sufrida por un país miembro del bloque, la Unión Europea amplió sus barreras no arancelarias, las cuales van desde el no cumplimiento de ciertos estándares de calidad del producto hasta denuncias por dumping.

Ya en el mes de la expropiación de Repsol YPF las exportaciones presentaron una variación interanual negativa, para luego presentar solo durante tres meses variaciones positivas leves. A partir de septiembre, los envíos de biodiesel al exterior se desplomaron, junto a la producción de la industria.

En un análisis más profundo, se verifica que la menor contracción de la producción se debe al incremento de la participación del mercado interno de biocombustibles, la cual creció significativamente desde 2009 hasta la fecha. Al analizar los datos se observa que el mercado interno creció de 176 toneladas en diciembre de 2009 a 67.275 toneladas en marzo de 2013, con un crecimiento máximo de 100.639 en julio de 2012. En dicho mes, hubo una modificación en la ley que establecía el uso de combustibles fósiles con una proporción de al menos un $5 \%$ de biocombustibles (B5) y se la reemplazó por otra que elevó a un $10 \%$ la proporción de biodiesel en los combustible.

\section{CONCLUSIONES}

El complejo oleaginoso argentino se encuentra en una fase avanzada de desarrollo. No solo se ha expandido fuertemente la producción primaria, especialmente de soja, sino también se han verificado eslabonamientos hacia atrás, adelante y a los lados. Debe señalarse que la existencia de ventajas comparativas en el cultivo de oleaginosas le ha dado a la Argentina un diferencial que ha sido aprovechado desde 1890 hasta 1930, fase en la que prevaleció el modelo agroexportador, para luego dar paso a la - fracasada - política de industrialización sustitutiva de importaciones. Hacia 1990, a pesar de la implementación del Consenso 
de Washington, nuevamente comenzó a darse impulso al sector agropecuario, ya que la apertura comercial impulsó el proceso de modernización de la actividad primaria. A partir de esa década, el complejo oleaginoso ya no solo presenta ventajas comparativas, sino también competitivas estáticas y dinámicas, ligadas al proceso de aprendizaje.

Como se mencionó, no solo hubo una mejora sustantiva en la producción primaria, sino también en la producción agroindustrial, principalmente en aceite de soja y otros derivados, y en la industria pesada, ya que el país es uno de los principales productores a nivel mundial de maquinaria agrícola. De igual forma, se han producido notables avances genéticos, lo que ha mejorado el rinde por hectárea y modificado el método de siembra.

El mejoramiento genético logró nuevas variedades decisivas en la expansión del área y el aumento de los rendimientos. El aporte de la biotecnología, con el clonado de genes y la transformación de plantas, fue determinante para el desarrollo del cultivo. Sin duda fueron las variedades transgénicas con tolerancia a glifosato (soja RR) -incorporadas a partir de 1996- las que dieron el mayor aporte al crecimiento del área. En pocos años se adoptaron casi un $100 \%$.

Esta implementación de nuevos cultivos trajo una expansión meteórica del cultivo. Por un lado, esta clase transgénica del poroto de soja puede ser implantada en zonas que hasta el momento resultaban inhóspitas para las variedades tradicionales, debido a su mayor tolerancia a climas cálidos y diferencias edáficas, tanto por su congráficación genética, como por el paquete químico y tecnológico que acompaña su siembra. Por otra parte, dentro de un contexto de gran inestabilidad económica y rigurosa presión impositiva, los productores vieron en esta oleaginosa de cotizaciones internacionales crecientes una gran oportunidad para incrementar la rentabilidad de sus áreas productivas, de manera menos arriesgada que la inherente a otros cultivos o a diversas explotaciones relacionadas con la cría de animales.

La expansión sorprendente experimentada por el sector agrario durante esta década ha sido generalizada, en lo referente a la producción física y a la demanda derivada de insumos cada vez más específicos y de mejor calidad. No obstante, para alcanzar los estándares mundiales de competitividad, las empresas debieron desarrollar un plan de inversiones que, en una primera etapa, estuvo dirigido a la incorporación de nueva tecnología y modernización de plantas. En una segunda etapa se privilegiaron las áreas comerciales, financieras y de infraestructura portuaria.

Una de las desventajas que presenta el complejo oleaginoso es que el alto precio internacional de la soja genera una tendencia al monocultivo, debido a su alta rentabilidad respecto a otros cultivos. Esta tendencia afecta directamente la fertilidad del suelo, que se resuelve mediante la aplicación de fertilizantes o con la rotación de cultivos, pero ambos factores tienden a disminuir la rentabilidad relativa. Otra gran desventaja es que no genera eslabonamientos adicionales a la cadena existente de cosecha, molienda y exportación mientras plantea una exagerada dependencia del mercado mundial.

Los principales problemas vinculados con el proceso de agricultura intensiva han tenido relación con los serios problemas de erosión y pérdida de fertilidad manifestados en las principales cuencas productivas de la Región Pampeana. El avance de la frontera agropecuaria y el proceso de artificialización de ecosistemas frágiles ameritan más que un proceso de intensificación, un sistema de complejización del espacio productivo, pobremente evaluado hasta la actualidad.

Por otra parte, si bien es cierto indicar que la siembra directa ha permitido disminuir o por lo menos desacelerar los procesos de erosión, la misma se ha llevado adelante y ha generado cambios sustanciales en el ecosistema, como la aparición de nuevas enfermedades, insectos y plagas, junto con aumentos de problemas de estructura en el perfil e incremento de la contaminación y aparición de resistencias en malezas e insectos.

Parte de estas desventajas, referidas al patrón de producción agrícola, pueden ser contrarrestadas con la producción de biocombustibles, disminuyendo al menos de manera parcial la dependencia a la 
volatilidad de los precios de los bienes primarios. Debe destacarse también la importancia de la producción de biocombustibles, ligados a la producción agrícola del país y las mejores introducidas en los últimos años.

Desde inicios de la presente década, los biocombustibles han adquirido un renovado interés a nivel mundial, lo cual se ha reflejado en una rápida expansión de su mercado. El objetivo primordial de los gobiernos para impulsar este desarrollo se encuentra en una mayor seguridad energética con una disminución de las importaciones de combustibles fósiles y el consecuente ahorro en divisas. En este contexto, las políticas gubernamentales han jugado y seguirán jugando un rol clave en el desarrollo de industria. No obstante, se debe considerar que estas políticas son costosas y de largo plazo y por lo tanto su costo-efectividad debe ser evaluado vis a vis de los objetivos de política establecidos tras la promoción de los biocombustibles. Luego, los gobiernos deben asegurar que los beneficiarios de dichas políticas son aquellos grupos objetivo que inicialmente se deseaba beneficiar con estas políticas de promoción (como pequeños agricultores, etc.).

Sin embargo, frente a la seguridad energética aparece como principal oponente el objetivo de seguridad alimentaria, ya que en el caso del biodiesel producido por Argentina, su feedstock es, por definición, un alimento, y dado el stock de soja un incremento en la producción de biodiesel disminuye la producción del cultivo para uso alimentario. Otro objetivo tradicional tras su promoción son las posibilidades que surgen para capturar oportunidades de desarrollo rural. No obstante, debe destacarse que a pesar de estos beneficios, han surgido preocupaciones respecto de su posible impacto sobre la seguridad alimentaria y sobre el medio ambiente.

Desde la perspectiva social, la promoción de desarrollo y empleo rural mejoran las condiciones de los pequeños agricultores y sus medios de vida. Es por esto que surge como uno de los objetivos primordiales para incentivar la producción de biodiesel. Por otra parte, las políticas de apoyo a dicho sector tienen mayor impacto sobre los multiplicadores sociales y económicos cuando involucran a pequeños productores. Esto plantea la necesidad de identificar modelos que equilibren la coexistencia de ambos sistemas de producción, para asegurar así una justa distribución de ganancias a lo largo de la cadena de valor e involucrando a pequeños productores.

En cuanto al desempeño del mercado en el mediano plazo, se estima que la producción aumentará rápidamente en los próximos años como consecuencia de la mayor demanda. Sin embargo, el ritmo de expansión dependerá en gran medida del precio del petróleo. En un escenario de precios del petróleo bajo (60 USD/ barril), el biodiesel y la mayoría de biocombustibles de primera generación no serán competitivos frente a los combustibles derivados del petróleo, mientras que en un escenario de altos precios del crudo (120 USD/barril), los biocombustibles se tornarán competitivos.

Así mismo, no solo debe considerarse el precio del petróleo en las proyecciones de producción de biodiesel, sino también la evolución de precios de los mercados agrícolas, debido a que en este tipo de producción el cultivo representa el $90 \%$ del costo del producto. En un escenario de precios de la soja a la alza, por el sostenido incremento de la demanda de los países emergentes - principalmente China e India - la suba de los costos directos de producción es un factor que atenta sobre la competitividad del producto.

Por último, en el marco de las restricciones impuestas por la Unión Europea, debe destacarse que un desafío importante al que se enfrenta Argentina es producir en concordancia con los estándares técnicos relevantes de los mercados importadores, a pesar del desarrollo de una exportadora exitosa.

En suma, la producción de biodiesel en el entorno del complejo oleaginoso argentino ofrece oportunidades para el logro de objetivos económicos, ambientales y sociales. Pese a la existencia de impactos negativos, existen oportunidades importantes, especialmente en la promoción de desarrollo local, que los gobiernos pueden abordar. 


\section{REFERENCIAS}

1. Anlló, G., Bisang, R. y Salvatierra, G. (2010). Cambios estructurales en las actividades agropecuarias. De lo primario a las cadenas globales de valor. Documentos de proyectos 50 , LC/W.350. CEPAL, Buenos Aires.

2. Becattini, G. (1989). Los distritos industriales y el reciente desarrollo italiano. Sociología del Trabajo, 5, 3-18.

3. Bisang, R. (2003). Posicionamiento del sector agroindustrial frente a la actual política económica. XX Jornadas de Perspectivas Agropecuarias 2002/2003. Rosario: Asociación Argentina de Economía Agraria.

4. Breschi, S. y Lissoni, F. (2003). Mobility and Social Networks: Localised Knowledge Spillovers Revisited. Centro di Ricerca sui Processi di Innovazione e Internazionalizzazione, Università Commerciale Luigi Bocconi, WP n.142.

5. Breschi, S. y Lissoni, F. (2001, marzo). Knowledge Spillovers and Local Innovation Systems: A Critical Survey. Liuc Papers, 84, 1-30.

6. Breschi, S. y Malerba, F. (2001). The Geography of Innovation and Economic Clustering: Some Introductory Notes. Industrial and Corporate Change, 10(4), 817-833.

7. Bresser-Pereira, L. (2006, enero-marzo). El nuevo desarrollismo y la ortodoxia tradicional. São Paulo em Perspectiva, 20(1), 7-29.

8. Civitaresi, H. y Granato, M., (2003). El complejo oleaginoso argentino: algunos factores determinantes de su desempeño exportador, Revista Argentina de Economía Agraria, Volumen VI (1).

9. Comisión Económica para América Latina y el Caribe (Cepal) (2001, marzo). Una década de luces y sombras. Notas de la Cepal, 15, 1-12.

10. De Obschatko, E. (1997). Articulación productiva a partir de los recursos naturales. Buenos Aires: Cepal.

11. Dufey, A. y Stange, D. (2011). Estudio regional sobre la economía de los biocombustibles en 2010: temas clave para los países de América Latina y el Caribe. Santiago de Chile: Cepal.

12. ExportaPymes (2002, noviembre). Economías regionales, una fuerte apuesta al mercado externo. Buenos Aires. Recuperado de http://exportapymes.com/article222.html

13. Fagerberg. J. (2003). The dynamics of technology, growth and trade: A Schumpeterian perspective. Working paper, 25, 3-27.

14. Fagerberg, J. y Verspagen, B. (2001). Technology - gaps, Innovation - diffusion and Transformation: an Evolutionary Interpretation. STI Working Papers, 1, 2-24.

15. Ferraro, C. (Comp.) (2010). Clusters y políticas de articulación productiva en América Latina. Buenos Aires: Cepal y Naciones Unidas.

16. Fogel, R. (2009, may). The Impact of the Asian Miracle on the Theory of Economic Growth. NBER Working Paper, 14967, 2-61.

17. Grossman, G. y Helpman, E. (1993, november). Endogenous Innovation in the Theory of Growth. NBER Working Paper, 4527, 23-44.

18. Gutman, G. (2000). Trayectorias y demandas tecnológicas de las Cadenas Agroindustriales en el Mercosur Ampliado: Oleaginosas. Proyecto Global Procisur/Bid, Procisur, Montevideo.

19. Hirschman, A. (1980). Auge y ocaso de la teoría económica del desarrollo. El Trimestre Económico, 47(4), 1055-1077.

20. Hirschman, A. (1961). Adopción de la teoría del crecimiento desequilibrado. En La estrategia del desarrollo económico. México: Fondo de la Cultura Económica. 
21. Kaldor, N. (1981). The Role of Increasing Returns, Technical Progress and Cumulative Causation in the Theory of International Trade and Economic Growth. Economie Appliquée, XXXIV, 593. 617. Krugman, P. (1998, april). The Role of Geography in Development. Annual World Bank Conference on Development Economics, Washington, D.C.

22. Llach, J. (1998). El crecimiento económico regional: el papel de los complejos productivos basados en los recursos naturales. Programa de Maestría en finanzas públicas provinciales y municipales. Universidad Nacional de La Plata, La Plata.

23. Marshall, A. (1890). Principles of economics. Londres: MacMillan.

24. Myrdal, G. (1956), Economic Theory and Under-Developed Regions. London: Gerald Duckworth \& Co. Ltd.

25. O'Connor, E. (2012a, july-september). Trade with China and Strategy in South American recent economic development. Brazilian Journal of Political Economy, 32 (3-128), 513-526.

26. O'Connor, E. (2012b, october). Paradigmas del desarrollo económico: el renacimiento del desarrollismo en Brasil y Argentina. Debates Latinoamericanos, 2 (20), 58-75.

27. Pietrobelli, C. y Rabellotti, R. (2005). Mejora de la competitividad en clusters y cadenas productivas en América Latina: el papel de las políticas. Washington, D.C.: Banco Interamericano de Desarrollo.

28. Prebisch, R. (1986). El desarrollo económico en América Latina y alguno de sus principales problemas. Desarrollo Económico,26(103). 5-63. Ramos, J. (1998, diciembre). Una estrategia de desarrollo a partir de los complejos productivos (clusters) en torno a los recursos naturales iuna estrategia prometedora? Revista de la Cepal, 66. 3-33.

29. Ricardo, D. (1973 [1817]). Principios de Economía Política y Tributación. México: Fondo de Cultura Economía.

30. Rodríguez, F. y Sachs, J. (1999, spetember). Why do Resource-Abundant Economies Grow more Slowly? Journal of Economic Growth, 4, 277-303.

31. Romer, P. (1994). The Origins of Endogenous-Growth. Journal of Economic Perspectives, 8(1), $3-22$.

32. Scialabba, E. (2013). Heterogeneidad estructural, brecha tecnológica e integración regional: estudio del caso Latinoamericano. III Congreso de la Internacional del Conocimiento, Foro Bienal de Estudios del Desarrollo 2013, Universidad de Chile, Santiago de Chile.

33. Scialabba, E. (2012). International trade and economic development in Latin America - A structuralist approach. Países de centro y periferia: lecciones de la historia económica y de la historia del pensamiento económico. Buenos Aires: CEFID-AR y ESHET.

34. Scialabba, E. (2011). Crecimiento endógeno, heterogeneidad estructural, brecha tecnológica y progreso técnico en América Latina. III Congreso Internacional en Desarrollo Económico y Calidad de Vida. Universidad La Gran Colombia, Bogotá.

35. Scialabba, E. (2010). Desarrollo económico e inserción internacional: industrialización e integración regional en América Latina. II Seminario Internacional de Desarrollo Económico y Calidad de Vida. Universidad La Gran Colombia, Bogotá.

36. Smith, A. (1958 [1776]). Investigación sobre la naturaleza y causas de la riqueza de las naciones. México: Fondo de Cultura Económica.

37. UN-Energy. (2007). Sustainable bioenergy: a framework for decision makers. Organización de las Naciones Unidas. Recuperado de http://esa.un.org/un-energy/pdf/susdev.Biofuels.FAO.pdf

38. Worldwatch Institute (2006). Biofuels for Transportation: Global Potential and Implications for Sustainable Agriculture and Energy in the 21st Century. Washintong, D.C.: Worldwatch Institute.

39. Young, A. (1928). Increasing Returns and Economic Progress. Economic Journal, 38, 527-42. 